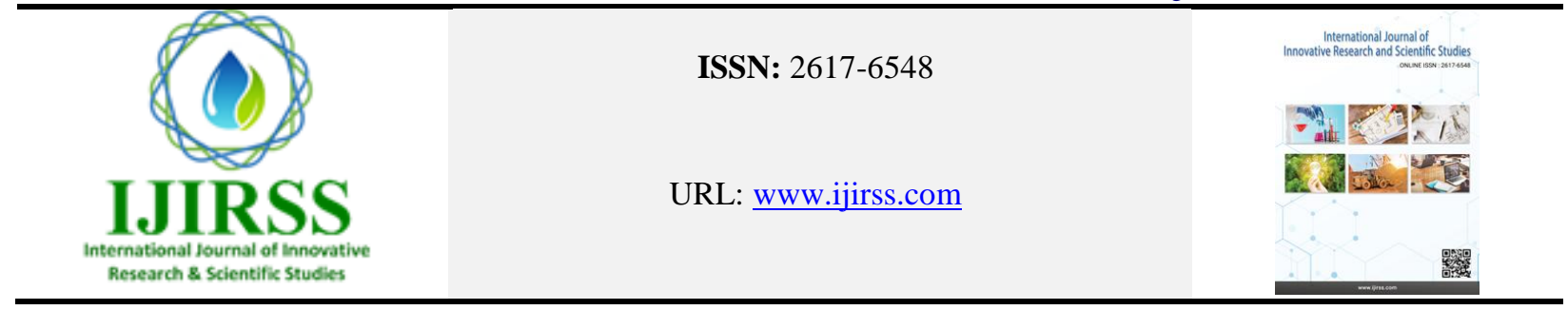

\title{
An Attitude to the Intellectual and Political thought of Seyed Jamaluddin Afghan
}

\author{
Muzhda Tabesh Noor \\ Department of History, Faculty of Social Sciences, University of Badakhshan, Badakhshan, Afghanistan \\ (Email:m.tabeshnoor@gmail.com)
}

\begin{abstract}
This study aimed to clarify the intellectual and political ideas of Sayed Jamaluddin Afghani, who is one of the most famous philosophers of the contemporary Islamic world and modern world and clarify his visions for the important issues of his era. In this paper, attempts have been made to find out analytical-descriptive approaches relying on library resources.The findings of the study indicated that Seyed Jamaluddin Afghani had a profound view on ideologies, movements, philosophical schools and social changes in the world, and his attitude to the important issues of his era was sociological and reformal, since in his first study, he comprehended the damages of Islamic communities and recommends worthwhile plans to escape the domination of the external powers and domestic tyranny that Islamic countries were struggling with. Seyed Jamaluddin, one of the celebrities of history has the most important position and particular virtue in terms of his role in regional developments, since he has had a glorious role in establishing freedom, equality and brotherhood in the history of relations between nations. An unknown Afghan thought possess from the prominent features of anti-tyranny, reformation of religious thought, convergence and Islamic ties. These parameters were indications of the titles that Seyed Jamal has given us as an important historical map.
\end{abstract}

Keywords: Pan-Islamism, Colonialism, Despotism, Islamic Civilization.

DOI: $10.53894 /$ ijirss.v2i3.20

Funding: This study received no specific financial support.

History: Received: 17 June 2019/Revised: 12 August 2019/Accepted: 23 August 2019/Published: 19 September 2019

Licensed: This work is licensed under a Creative Commons Attribution 4.0 License $(\mathrm{ccc}) \mathrm{EY}$

Competing Interests: The author declares that there are no conflicts of interests regarding the publication of this paper.

Transparency: The author confirms that the manuscript is an honest, accurate, and transparent account of the study was reported; that no vital features of the study have been omitted; and that any discrepancies from the study as planned have been explained.

Ethical: This study follows all ethical practices during writing. 


\title{
نعرشى به انديشه اى فكرى و سياسى سيد جمال الاين افغان
}

\author{
مزّده تابش نور \\ دبيإزتمنت تاريخ، دانشكدة علوم اجتماعى، د/نشكاه باخثان، بلخشان، افغانستان
}

خلاصه

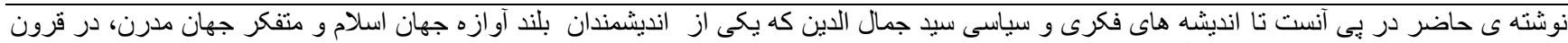

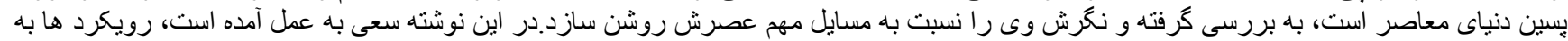

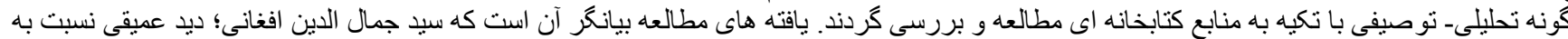

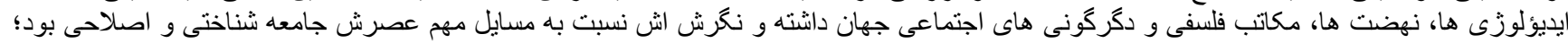

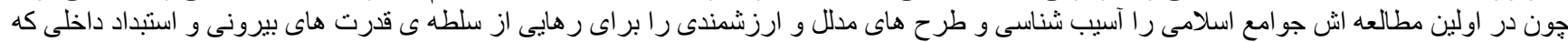

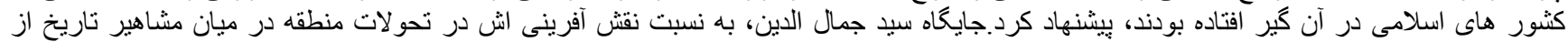

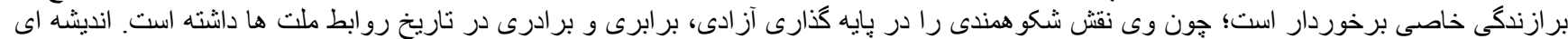

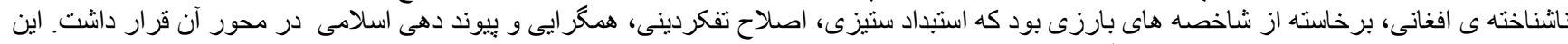

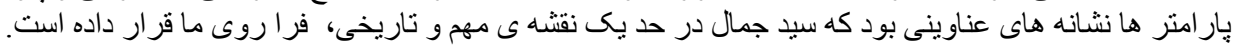

كلمات كلي!ى : پان اسلاميزم، استعمار، استبداد، تمدن اسلامى

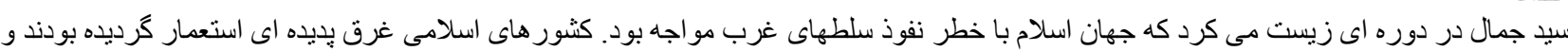

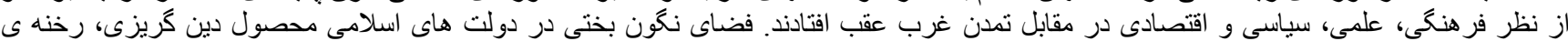

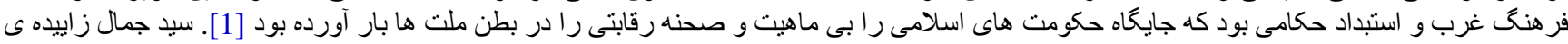

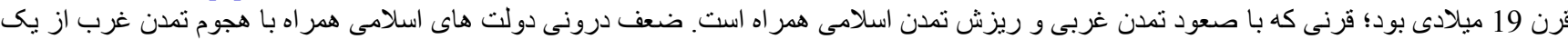

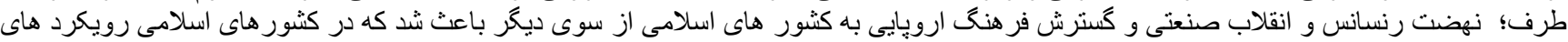

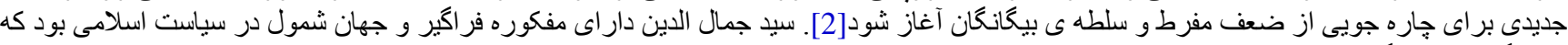

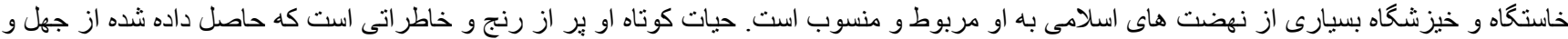

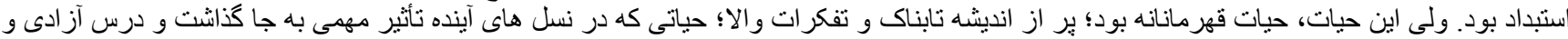

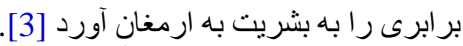

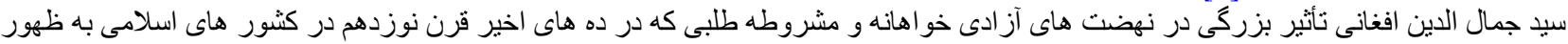

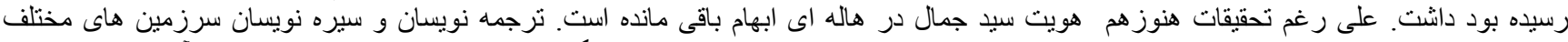

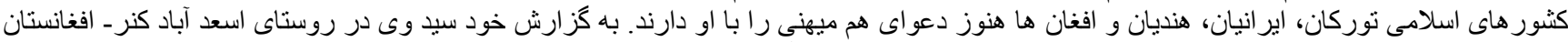

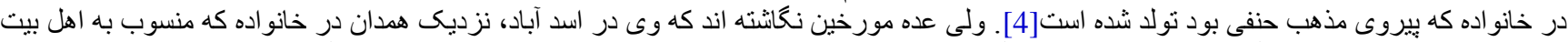

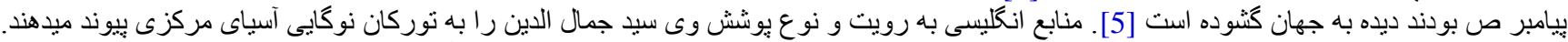

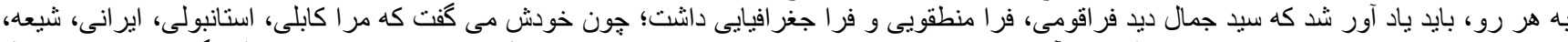

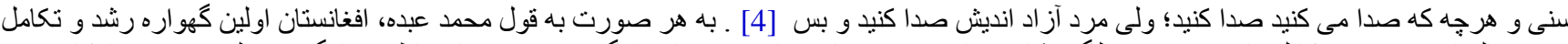

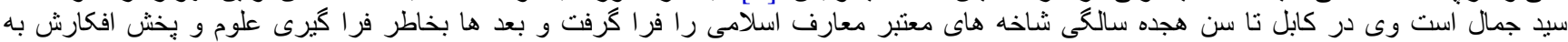

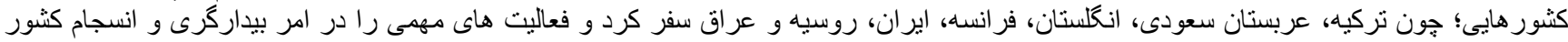

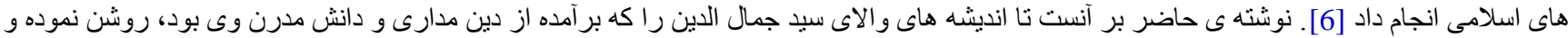

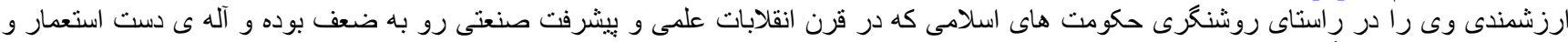

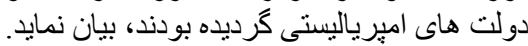

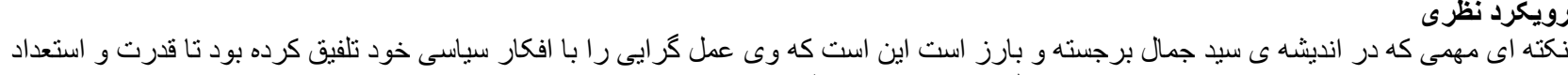

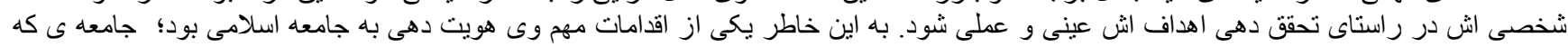

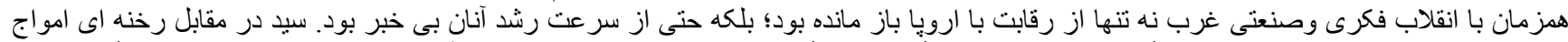

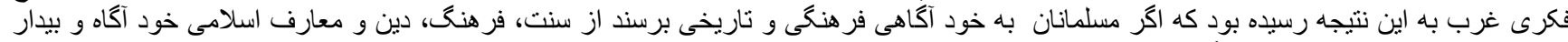

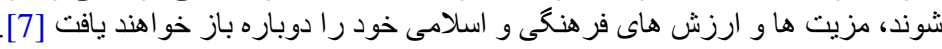

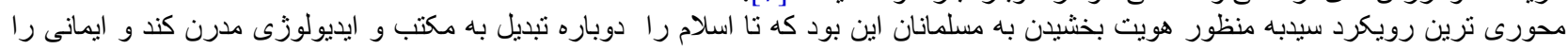

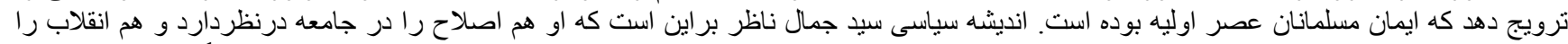

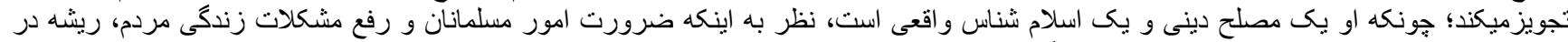

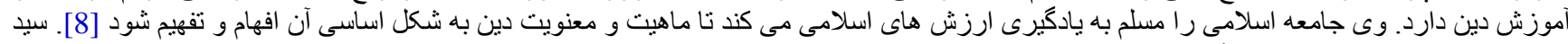

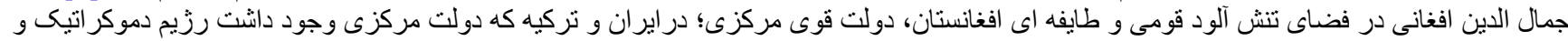

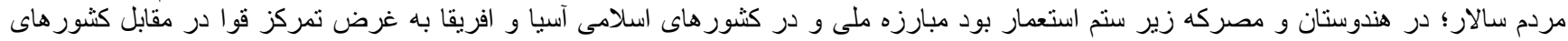

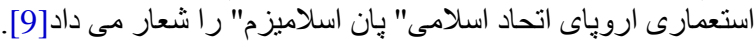

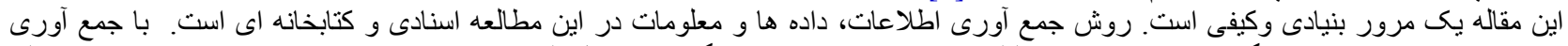

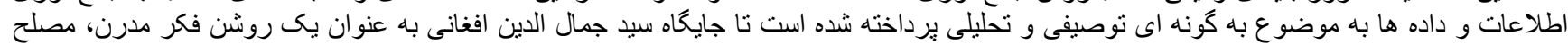

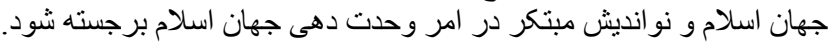




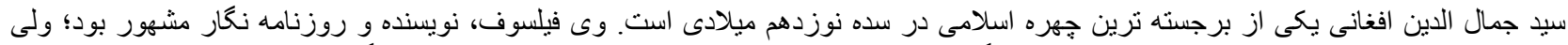

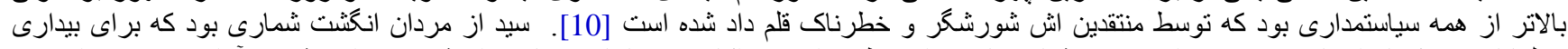

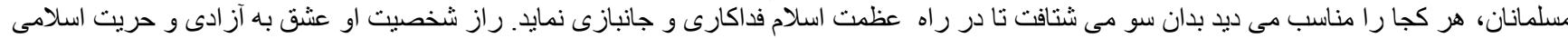

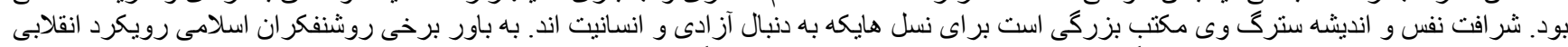

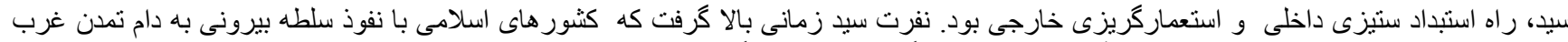

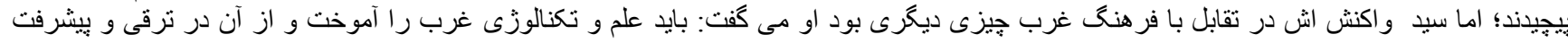

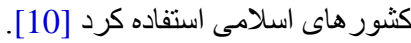

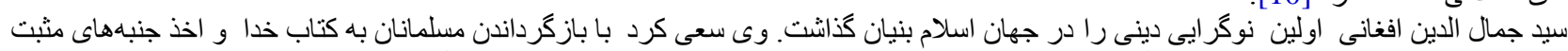

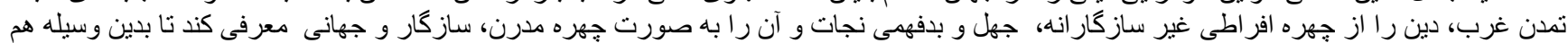

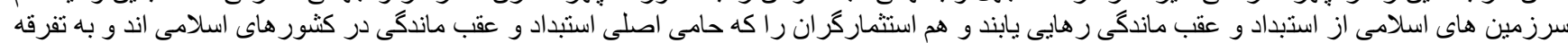

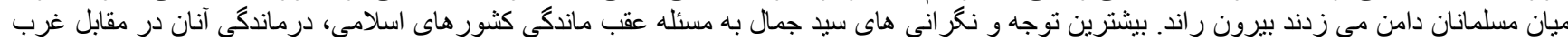

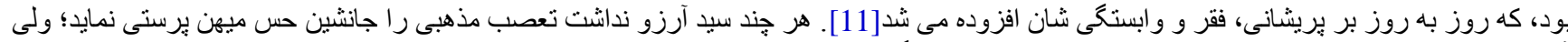

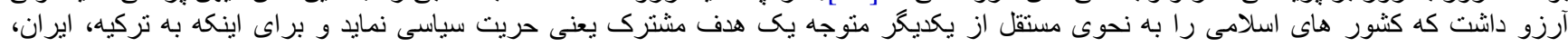

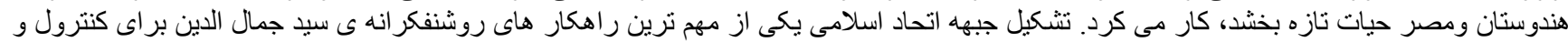

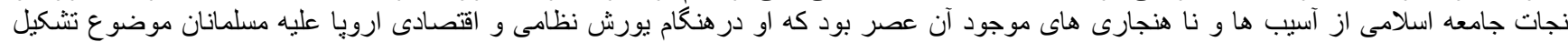

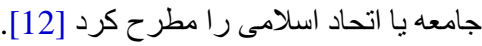

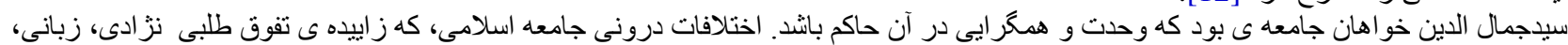

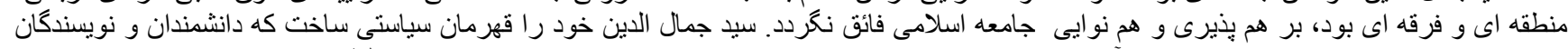

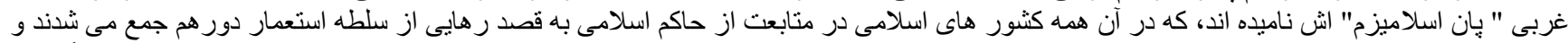

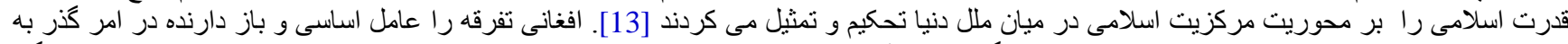

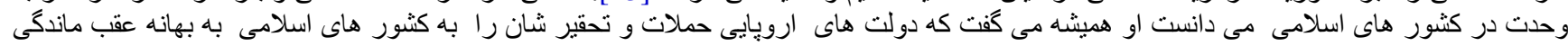

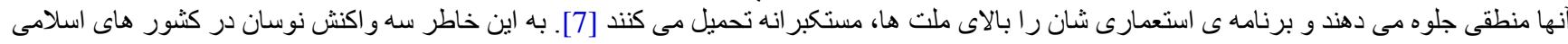

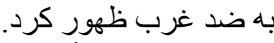

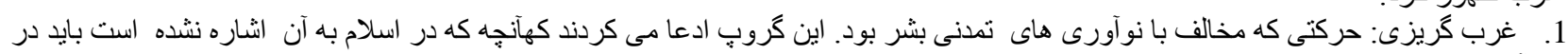
مخالفت به آن بسيج شُد.

2.

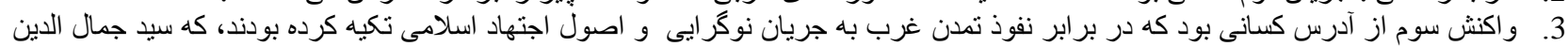

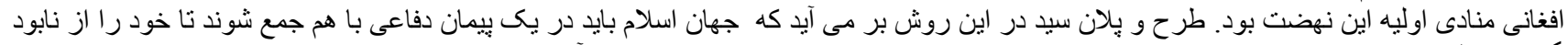

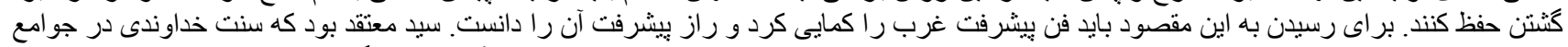

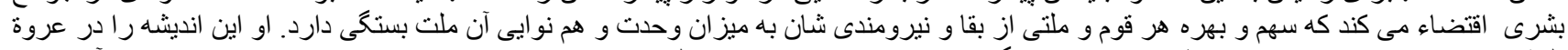

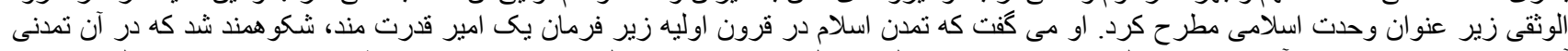

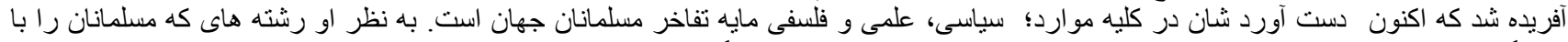

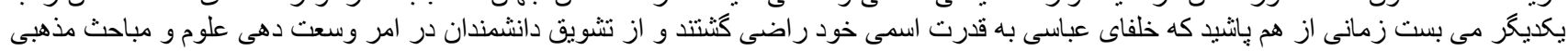

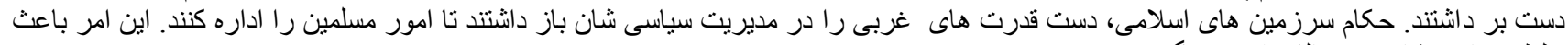

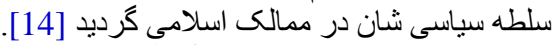

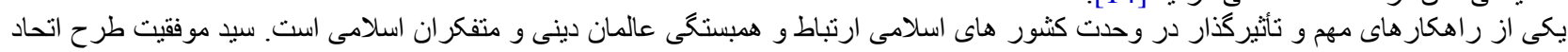

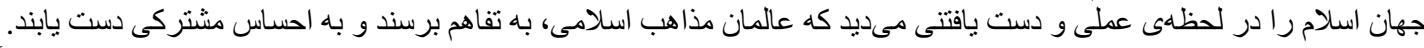

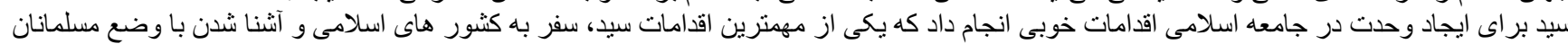

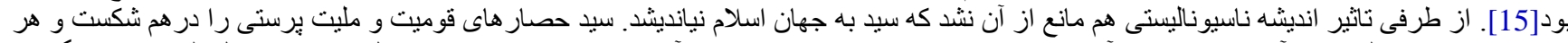

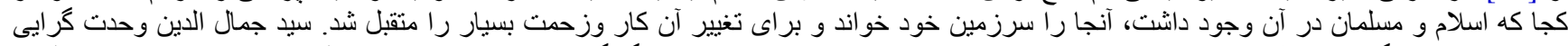

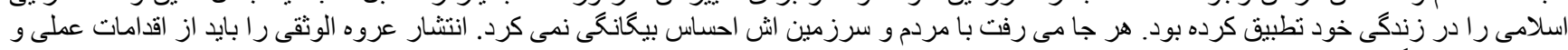

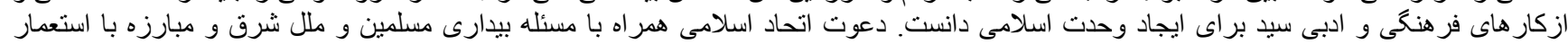

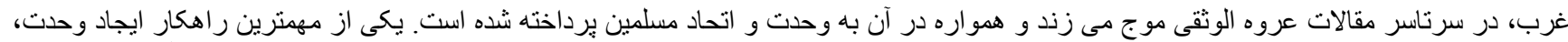

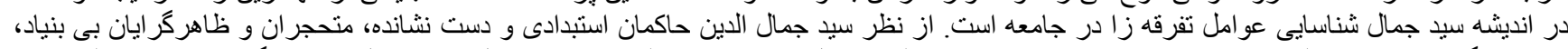

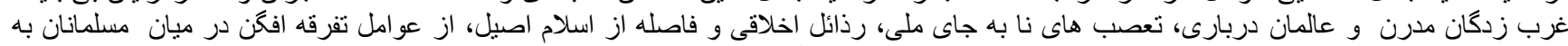

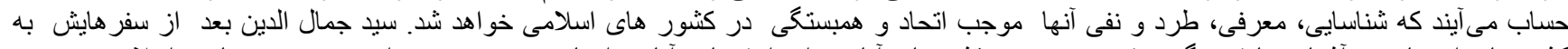

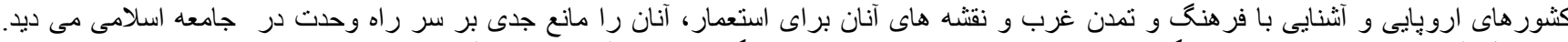

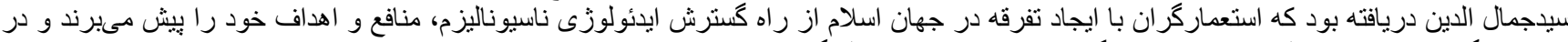

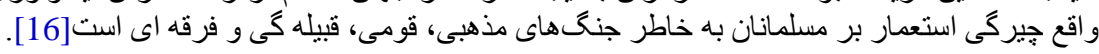

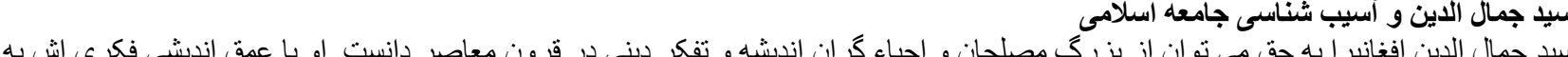

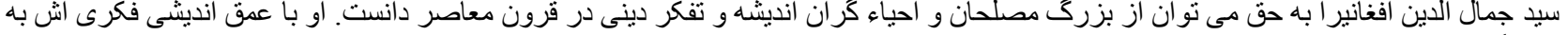

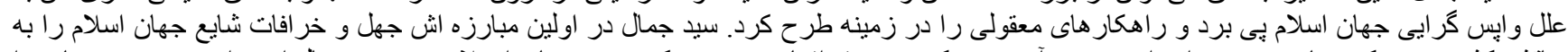

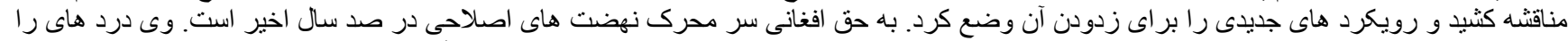

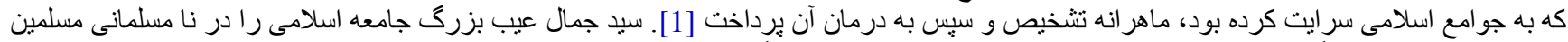

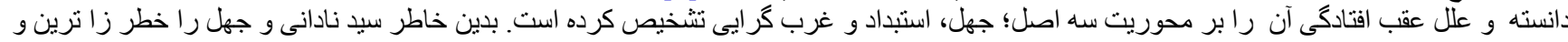

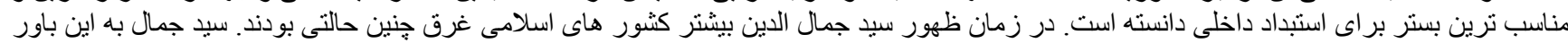

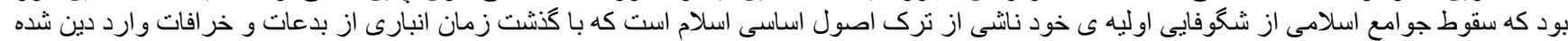

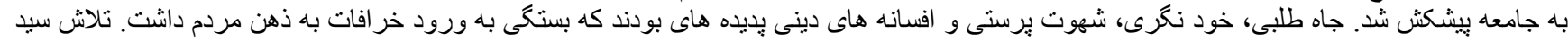

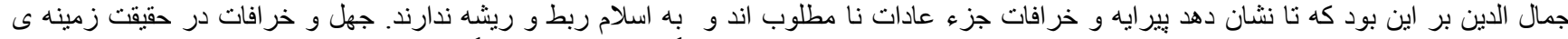

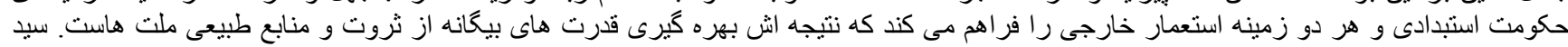

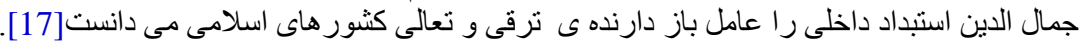




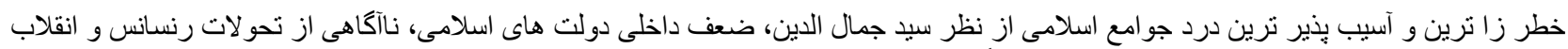

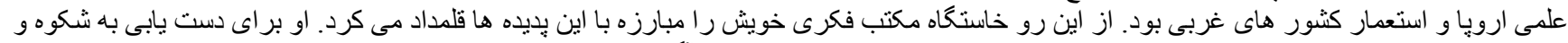

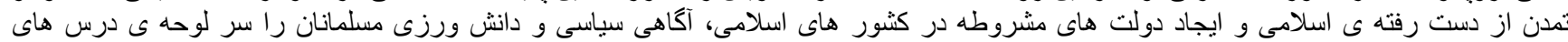

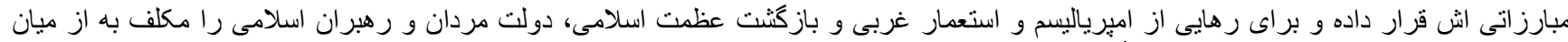

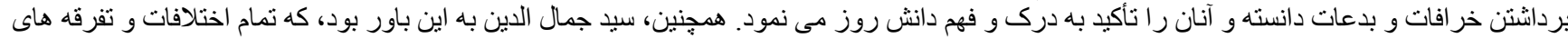

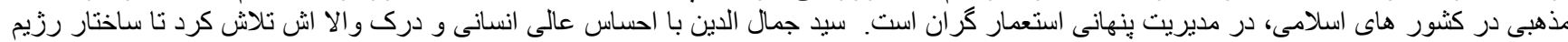

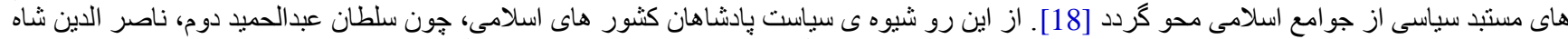

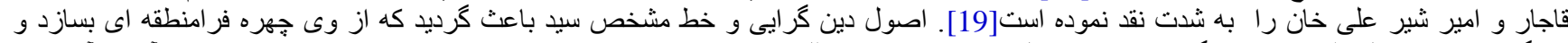

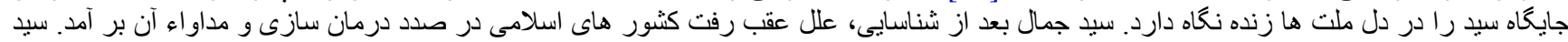

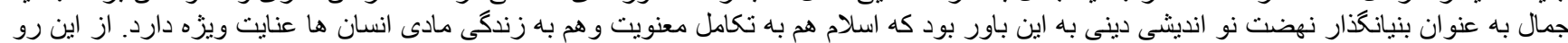

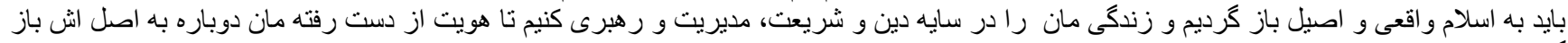

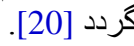

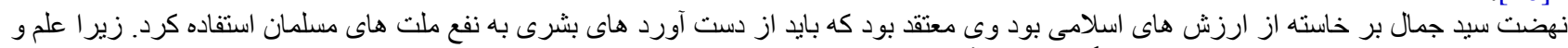

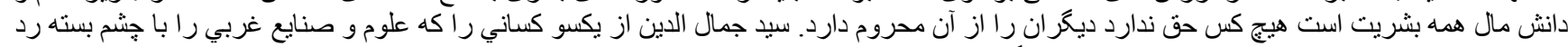

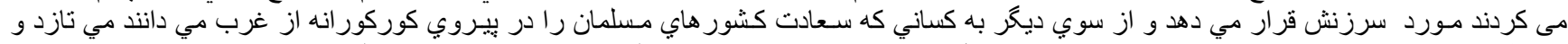

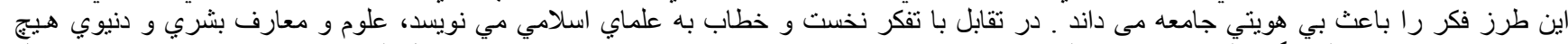

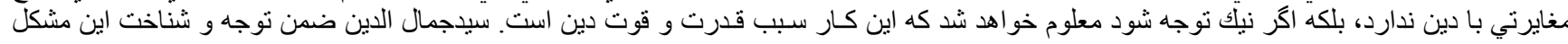

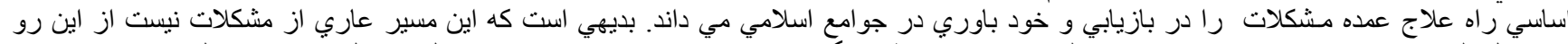

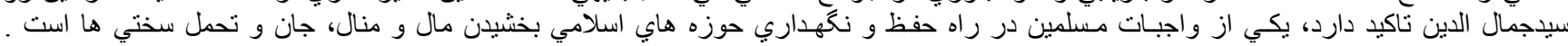

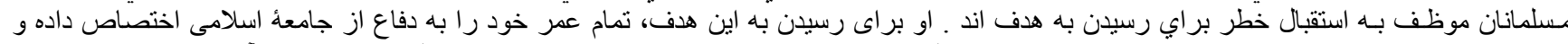

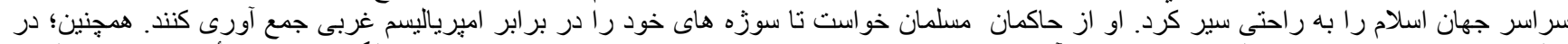

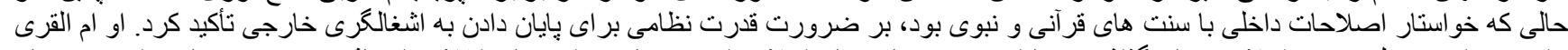

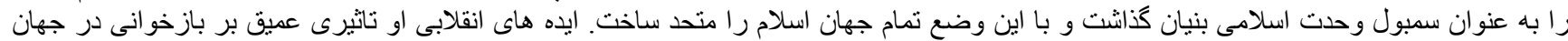

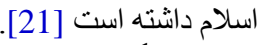

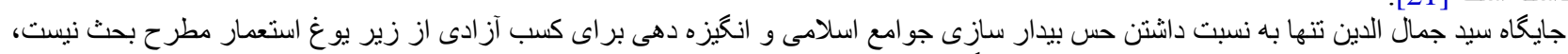

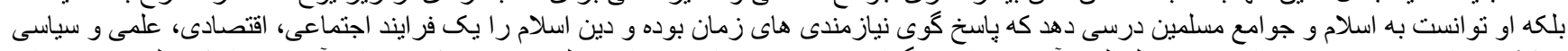

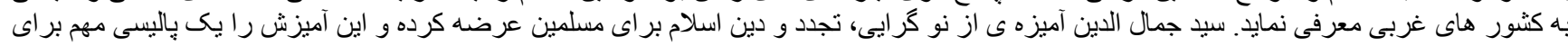

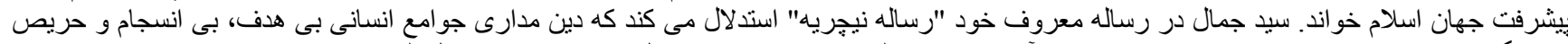

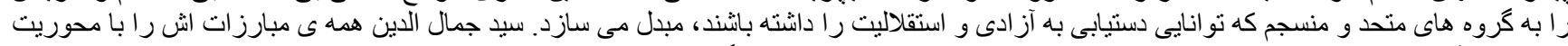

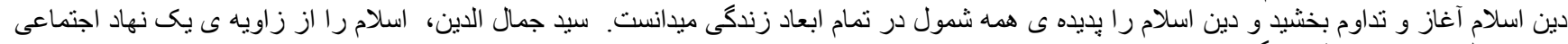

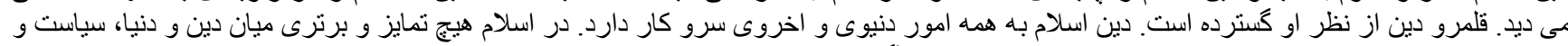

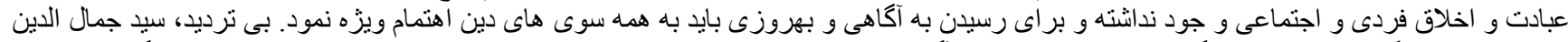

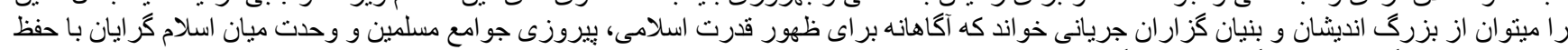

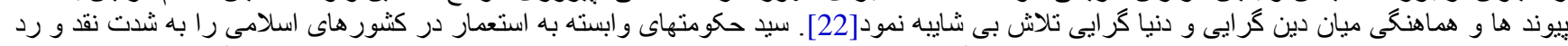

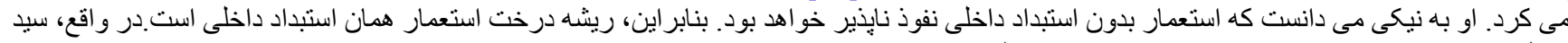

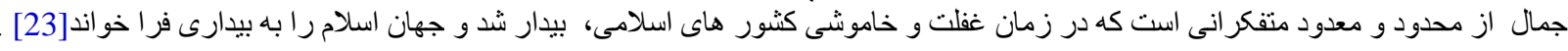

نهضت فكرى و مدل اصلاحى سيا جمال

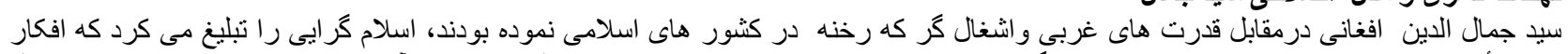

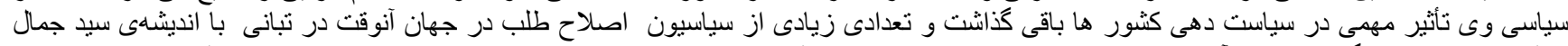

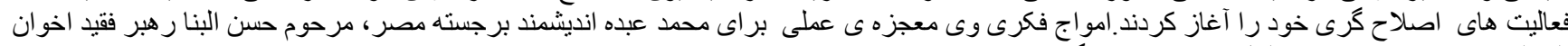

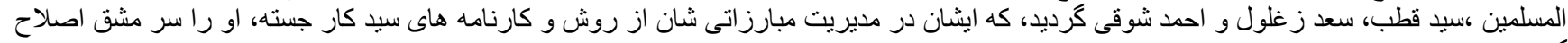

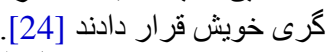

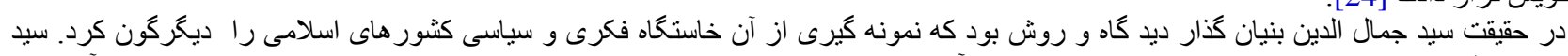

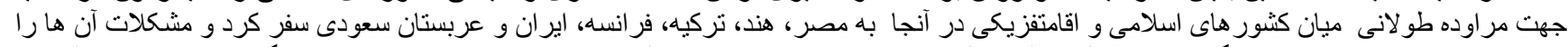

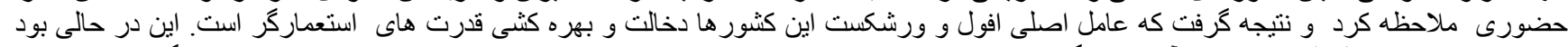

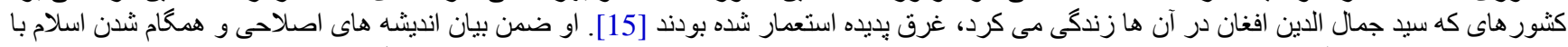

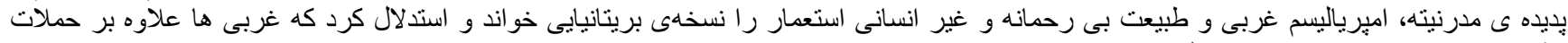

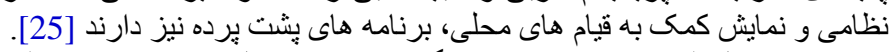

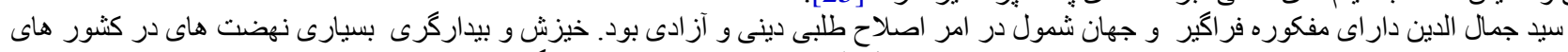

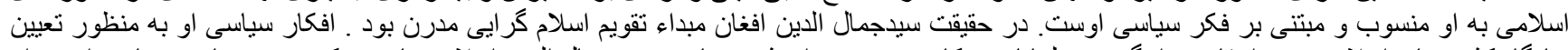

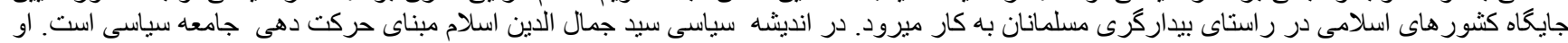

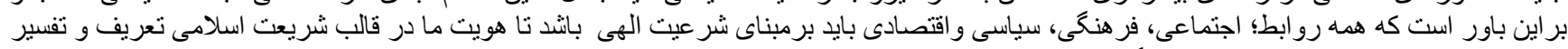

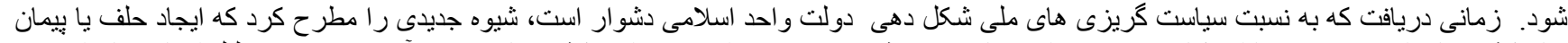

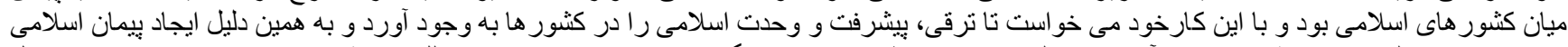

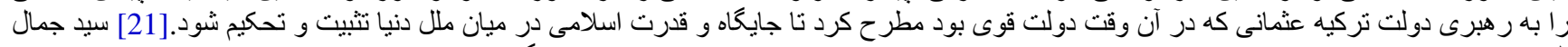

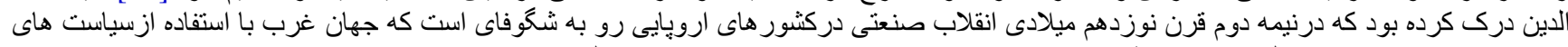

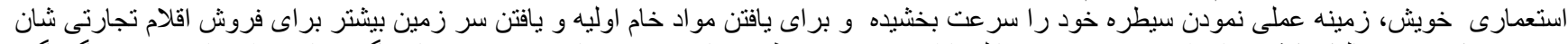

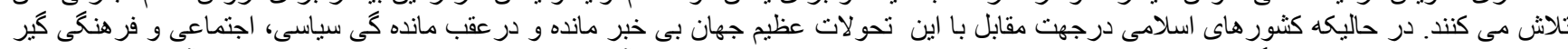

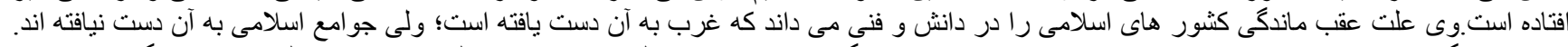

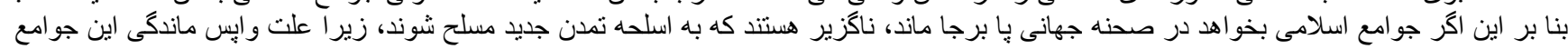

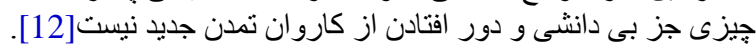




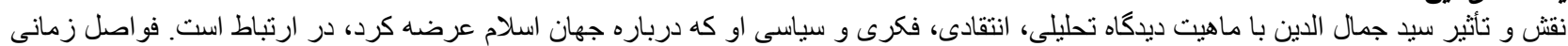

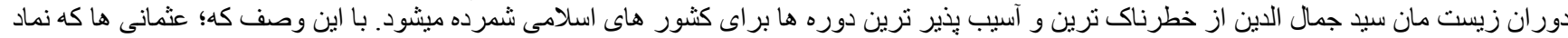

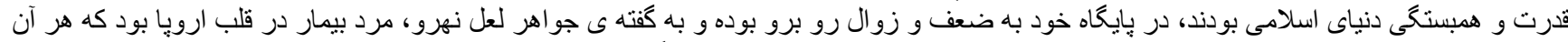

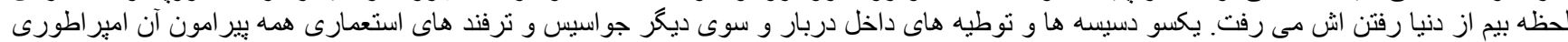

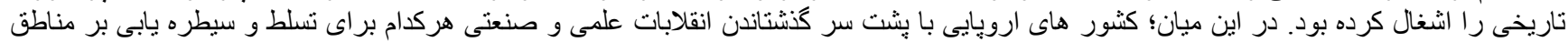

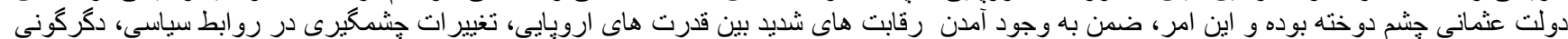

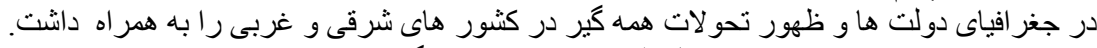

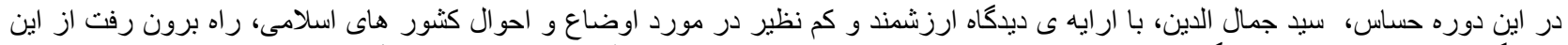

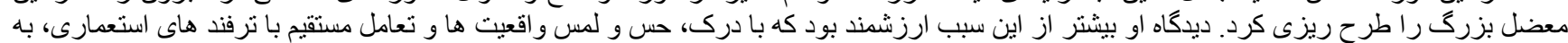

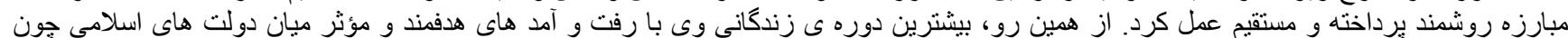

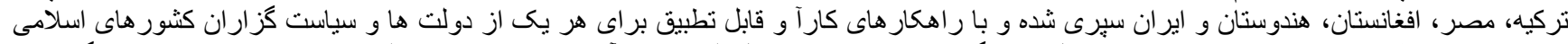

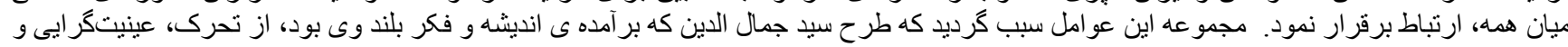

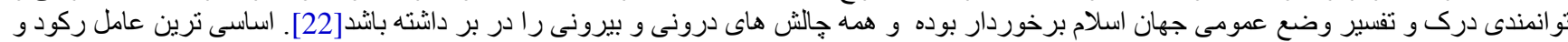

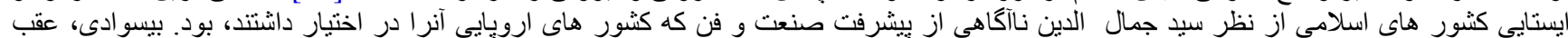

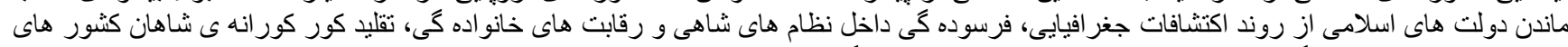

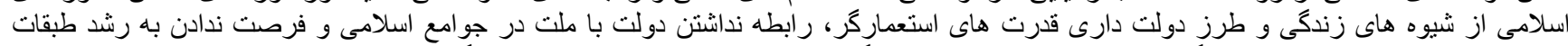

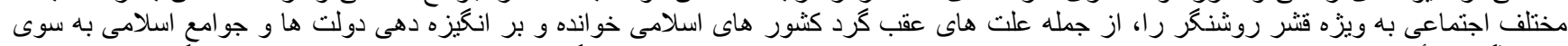

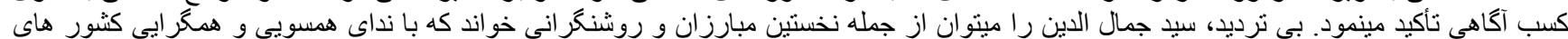

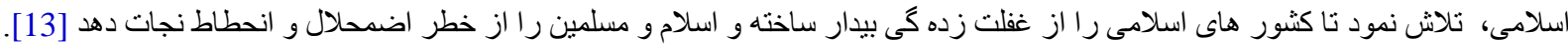

نتيجه كيرى

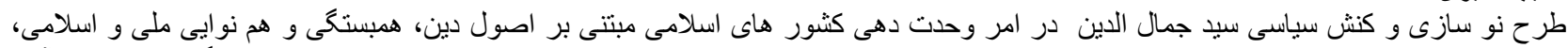

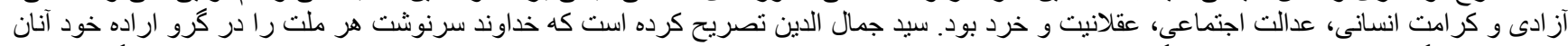

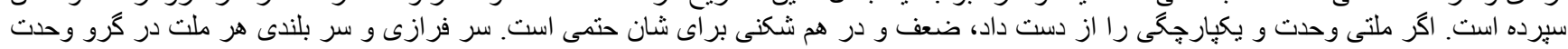

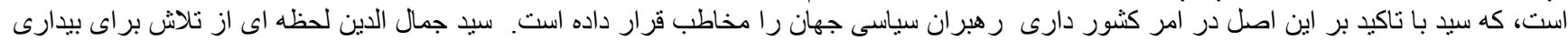

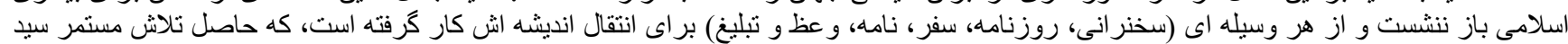

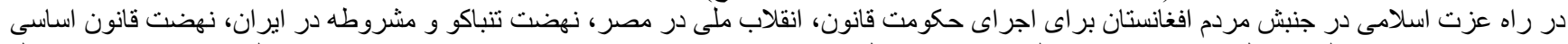

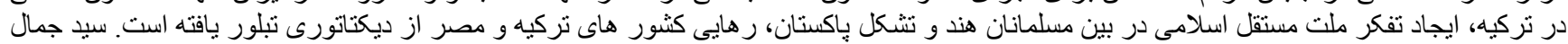

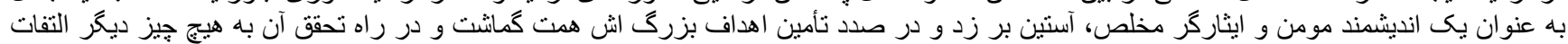

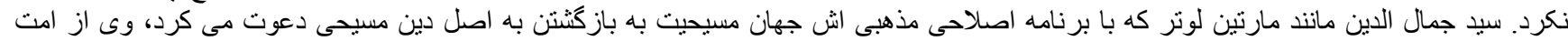

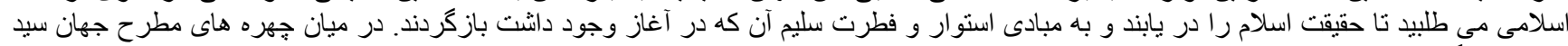

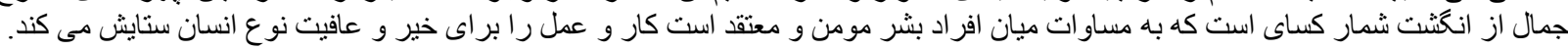

\section{References}

[1] A. L. John, "Jamal al-Din al- in the Islamic world: Past and present. Retrieved from http://www.oxfordislamicstudies.com/article/opr/t243/e8 " 2019.

[2] H. Abdul-Hadi, "Afghānī on the decline of Islam, Die Welt des Islams, new series," vol. 13 pp. 121-125, 1971.

[3] R. Rasool, History of the Afghan press; From Shams Al-Nahar to the Republic. Kabul: Maiwand Publications, 2008.

[4] F. Fazel, "Sayed Jamaluddin Afghani and the Elites of Afghanistan," Ariana Afghanistan Online, pp. 10 1-8, 2017.

[5] M. B. Linam, "A study of the personality of Seyyed Jamaluddin and his role in Awakening muslim thoughts," pp. 1-4, 2011.

[6] M. M. Seyyed, Seyyed Jamaluddin Asadabadi and the Eastern Awakening vol. 18 Tehran: Islamic Culture Publishing Office, 1991.

[7] N. R. Keddie, "Pan-Islam as Proto-Nationalism," The Journal of Modern History, vol. 41 pp. 17-28., 1969.

[8] J. P. Montada, "Al-Afghani, a case of religious unbelief, studia Islamica," vol. 100 pp. 203-220, 2005.

[9] Qalachi, Hakim Mashreq Seyyed Jamaluddin Afghan vol. 5. Kabul: Maiwand Publishing House, 2012.

[10] Modarressi, Seyyed Jamaluddin and his thoughts. Tehran: Amir Kabir Publishing, 2002.

[11] M. L. Kenny, "Al-Afghānī on types of despotic government," Journal of the American Oriental Society, vol. 86, pp. 19-27, 1966.

[12] Majid, "Intellectual components in the social thought of Seyed Jamaluddin Afghani," Islamic and Social Sciences Quarterly, vol. 2, pp. 115-140, 2010.

[13] Y. Mehdi and S. Sajjad, "A look at the thoughts of Seyyed Jamaluddin Afghani," Farhangian Scientific and Research Journal, vol. 5, pp. 124-134, 2008.

[14] R. Mohammad, Reflections on the political behavior of Seyyed Jamaluddin, Translator, Tabraian, Safaeddin vol. 56 Tehran: Safflower Publications, 1987.

[15] M. Ghulam, Afghanistan in the path of history. Kabul: Government Press, 1346.

[16] Bahram, "A study of the thoughts of Seyyed Jamaluddin Afghani," Research Institute for Cultural and Social Studies and the Ministry of Science of Iran, vol. 3, pp. 34-46, 2009.

[17] B. N. Mohammad, "Sayed Jamaluddin Afghan and the main cause of backwardness of Islamic societies," Ariana Afghanistan Online, vol. 6, pp. 6-8, 2016.

[18] Seyyed Jamaluddin Asadabadi, "Islamic encyclopedia (Encyclopedia of Characters)," pp. 1-3, 2015.

[19] R. Matthee, "Jamal al-Din al-Afghani and the Egyptian national debate," International Journal of Middle East Studies, vol. 21, pp. 151-169, 1989.

[20] J. Hirano, "Historical formation of Pan- Islamism: modern Islamic reformists project for Intra-Umma alliance and intermadhahib rapprochement,", Kyoto Working Papers on Area Studies: G-COE Series, No.102008. 
[21] W. Omid, Sayed Jamaluddin Afghan as a social reformer in Islamic world vol. 7: Andhra University: Vishakapatnam, Department of English, 2017.

[22] B. Morteza, "The initiator of the Islamic Awakening from the perspective of the Times. Retrieved from http://www.mouood.org/arabic/item," 2013.

[23] D. A. Nasir, "Sayyid Jamaluddin Afghani's theories in the field of education," Journal of Erfan, pp. 5-1, 1990.

[24] M. Caliskan, "Jamaluddin Al Afghani: The roots of revivalism. Retrieved from https://www.academia.edu/514993/Ideas_and_Activities_of_Sayid_JamalaldinAfghani," 2010.

[25] M. Tanvir, "Sayed Jamaluddin Afghan: Life \& work," Kabul, ministry of Information and culture of Islamic Republic of Afghanistan, 2007. 